\title{
Monozygotic twinning associated with assisted reproductive technologies: a review
}

\author{
K I Aston ${ }^{1,2,3}$, C M Peterson ${ }^{4}$ and D T Carrell ${ }^{1,2,3,4}$ \\ ${ }^{1}$ Andrology and IVF Laboratories, Departments of ${ }^{2}$ Surgery, ${ }^{3}$ Physiology and ${ }^{4}$ Obstetrics and Gynecology, University \\ of Utah School of Medicine, Salt Lake City, Utah 84108, USA \\ Correspondence should be addressed to D T Carrell at Andrology and IVF Laboratories, University of Utah School of Medicine, 675 \\ Arapeen Drive, Ste 205, Salt Lake City, Utah 84117, USA; Email: douglas.carrell@hsc.utah.edu
}

\begin{abstract}
Twin birth rates have increased markedly in developed countries since the 1970s for two primary reasons: increasing maternal age and the advent and increasing use of fertility treatments. In addition, monozygotic (MZ) twin pregnancies have been reported to occur at a significantly higher rate following assisted reproductive technologies (ART) procedures compared with the natural incidence. Twin pregnancies are of concern due to a dramatically increased risk of associated complications. Monozygotic twin pregnancies carry a $\mathbf{1 0 - 2 0} \%$ risk of twin-twin transfusion syndrome, and monoamniotic monochorionic twins are additionally at risk for cord entanglement. While the mechanisms and contributory factors for dizygotic twinning are well established, very little is known about the mechanisms involved in MZ twinning or the factors that contribute to its occurrence. In this review, we will discuss a number of potential mechanisms involved in $\mathrm{MZ}$ twinning and explore factors that may be contributing to the increased incidence of ART-associated MZ twins. An improved understanding of the factors that contribute to increased MZ twinning associated with ART will help to elucidate the poorly understood mechanisms involved in the process and will further aid in reducing the overall incidence of multiple pregnancies with their associated risks following ART procedures.

Reproduction (2008) 136 377-386
\end{abstract}

\section{Introduction}

In humans, twins generally result from one of two scenarios. Dizygotic (DZ) twins result from the ovulation and fertilization of two oocytes with the resultant twins sharing the same genetic relationship as common siblings. Monozygotic (MZ) twins, on the other hand, are the result of a single fertilized embryo splitting at some stage of early embryo development to form two separate embryos derived from one zygote. DZ twinning is associated with increased maternal serum folliclestimulating hormone associated with maternal genetics (White \& Wyshak 1964), age (Beemsterboer et al. 2006), parity (Rao 1978), and other factors (Hall 2003). The occurrence of DZ twinning varies widely between populations ranging from about 6/1000 in Asia to $10-20 / 1000$ in the United States and Europe and as high as 40/1000 in Africa (Hall 2003).

While the genetic contribution to DZ twinning is well established, genetic associations with the occurrence of MZ twins have been described infrequently, and penetrance for this trait seems to be extremely low (Parisi et al. 1983, Steinman 2003, Hamamy et al. 2004). Monozygotic twin occurrence is universally about $0.4-0.45 \%$ following non-stimulated in vivo conception (MacGillivray 1986,
Derom et al. 1987). The universally low incidence of $M Z$ twinning independent of environmental factors has been suggested to support the notion of a genetic rather than an environmental contribution (Bortolus et al. 1999). Overall, MZ twin pregnancies account for $\sim 30 \%$ of all naturally conceived twin pregnancies.

With the relatively recent advent and growing popularity of assisted reproductive technology (ART) procedures, the occurrence of both DZ and MZ twins has increased. Monozygotic twinning following in vitro fertilization (IVF) was first reported in 1984 (Yovich et al. 1984), and since that time numerous studies have demonstrated an increased occurrence of $M Z$ twins associated with ART procedures. Following 127977 ART procedures in the United States in 2004, 32\% of live birth deliveries were twin or higher order pregnancies (Wright et al. 2007). In Europe, summaries of 365103 ART cycles in 2003 indicated that twin and triplet pregnancies accounted for $23 \%$ of all deliveries (Andersen et al. 2007).

The United States and European summaries do not include data on the zygosity of twin births, but other published reports indicate that $M Z$ twinning rates following ART procedures are between two and twelve times higher than the natural occurrence of $0.4 \%$ 
(Edwards et al. 1986, Blickstein et al. 1999, 2003, Sills et al. 2000a, Alikani et al. 2003), and several cases of MZ triplets derived from ART procedures have also been reported (Ghulmiyyah et al. 2003, Risquez et al. 2004, Unger et al. 2004, Henne et al. 2005, Faraj et al. 2007, Yanaihara et al. 2007). A population-based study analyzing data provided by the British Human Fertilization and Embryology Authority representing 15644 cycles involving single embryo transfers and 1104 live births found the rate of multiple pregnancies among live births to be $2.3 \%$ (Blickstein et al. 2003). In many studies, the rate of $M Z$ twinning is erroneously based only on the number of monochorionic twins detectable by ultrasound, and dichorionic twins are inaccurately labeled as DZ. This undoubtedly results in underrepresentation of the actual rate of $M Z$ twinning in many cases (Wenstrom et al. 1993, Blickstein 2005).

Following ART procedures, DZ twin occurrence is higher as a result of ovarian stimulation protocols and multiple embryo transfers. The reasons for increased $M Z$ twinning frequency associated with ART techniques are less well defined; however, ovarian stimulation, zona manipulation including intracytoplasmic sperm injection (ICSI) and assisted hatching ( $\mathrm{AH})$, maternal age, and factors associated with in vitro embryo culture may contribute as discussed below.

\section{Complications in twin pregnancies}

The occurrence of $M Z$ twins is of clinical importance because of the increased complications associated with twin pregnancies in general as well as $M Z$ twins specifically. Twin pregnancies are characterized by an increased incidence of both fetal and maternal complications. Fetal complications include restricted growth as well as increased incidence of fetal loss, pre-term delivery, and perinatal loss. Mothers carrying twins more frequently suffer pregnancy-associated nausea and vomiting, gestational hypertension, and pre-eclampsia than those carrying singletons (Rao et al. 2004).

Placental arrangement is an important factor in outcome of $\mathrm{MZ}$ pregnancies, as occurrence of complications depends largely on the degree of placental sharing. The placental architecture of $M Z$ twins, which can vary from completely separate amniotic and chorionic sacs to a single amniotic and chorionic sac shared by both twins, indicates the embryonic stage at which the embryo splits. If the embryo divides prior to embryonic cell differentiation (within the first 4 days following fertilization), the resultant fetuses will be dichorionic diamniotic (DCDA). If the inner cell mass (ICM) divides during the pre-implantation blastocyst stage, the twins will be monochorionic diamniotic (MCDA), and if the division occurs in the postimplantation blastocyst period, monochorionic monoamniotic (MCMA) twins result (Hall 2003). DCDA conformation accounts for $\sim 25-30 \%$ of $M Z$ twin pregnancies, MCDA occurs in 70-75\% of MZ twin pregnancies, and MCMA in $1-2 \%$ of liveborn $M Z$ twins (Hall 2003). The great majority of DZ twins display a DCDA placental arrangement; however, it should be noted that an MCDA arrangement has been observed in DZ twins (Souter et al. 2003).

The incidence of fetal complications is similar between DZ twins and dichorionic MZ twins; however, monochorionic twins are susceptible to additional complications. The majority of monochorionic twins have vascular anastomoses, and this shared blood supply can result in twin-twin transfusion syndrome, a condition characterized by unequal sharing of the maternal blood supply, which results in asymmetrical fetal growth and fetal mortality in $80 \%$ or more of untreated cases (Fieni et al. 2004). The syndrome is estimated to occur in $10-20 \%$ of monochorionic twin pregnancies (Harkness \& Crombleholme 2005). MCMA twin pregnancies carry even greater risk due to the potential for umbilical cord entanglement (Dickinson 2005).

A study of the natural course of $M Z$ twin pregnancies evaluated pregnancy outcomes of $M Z$ twins in a historical cohort of 651 twin pairs born between 1907 and 1938. In this group, perinatal mortality was $27.7 \%$ for monochorionic twins and $15.8 \%$ for dichorionic twins, and the risk of perinatal mortality was associated with low birth weight and pre-term delivery (Hack et al. 2006). Owing to the scarcity of data reporting chorionicity of ART-derived $M Z$ twins, the relative occurrence of each type is unclear, but a recent report of 74 ART-derived MZ pregnancies of known chorionicity indicated $\sim 10 \%$ were DCDA, 53\% were MCDA, and an alarming $37 \%$ were MCMA (compared with $1-2 \%$ of in vivo produced MZ twins; Alikani et al. 2003). Derom et al. (1987) reviewed a much smaller number of $M Z$ twins and found only $46 \%$ of $M Z$ twins resulting from ovarian stimulation were monochorionic compared with $63 \%$ of controls, but the difference was not significant. The same group later reported an increased incidence of monochorionic $M Z$ twins following ovarian stimulation of $80 \%$ compared with $64 \%$ in controls (Derom et al. 2006). One study has been cited as reporting a significantly lower incidence of monochorionic twins following ovarian stimulation and IVF compared with spontaneously conceived twins, but zygosity of dichorionic twins was not characterized in this study (Putterman et al. 2003) so the relative reduction in monochorionic twins is most likely the result of a disproportionate increase in DZ twin pregnancies.

\section{Proposed mechanisms for MZ twinning}

Surprisingly little is known about the mechanisms or risk factors involved in early embryonic development that give rise to $M Z$ twins; however, a number of theories have been proposed. It is clear based on the various placental 
configurations observed in $M Z$ twins that timing of the twinning event as well as the mechanism involved can vary (Scott 2002). A major limitation in understanding MZ twinning mechanisms is the lack of animal models for study. The only naturally occurring animal model to produce $\mathrm{MZ}$ offspring on a regular basis is the armadillo, which produces identical quadruplets resulting from two binary fissions of a single fertilized oocyte (Blickstein \& Keith 2007). It has been proposed that these fission events are the result of delayed post-fertilization implantation in the armadillo. Following breeding, the embryo develops to hatching blastocyst and then undergoes a period of developmental arrest. After several months of arrested development, the embryo resumes growth, the epiblast undergoes the two binary fissions, and implantation occurs (Enders 1962, 2002a).

The hypothesis that embryo fission occurs as a result of delayed implantation does not reconcile the absence of $M Z$ twin formation in other species that undergo embryonic diapause; however, there may be some conserved mechanisms common to armadillo and some cases of human MZ twinning. The possibility of some human $M Z$ twins arising from a late splitting event similar to the armadillo is supported by the observation of a partially split embryonic shield in a day 15 rhesus monkey embryo (Enders 2002b).

Monozygotic twinning has been induced experimentally in a number of species by a variety of mechanisms. Early in the twentieth century, experiments in fish demonstrated slowing of development in early fertilized embryos by either lowering incubation temperature or reducing available oxygen resulted in increased incidence of MZ twins (Stockard 1921). Likewise, hypoxic embryo culture in mice and delayed fertilization in rabbits has been shown to induce $M Z$ twinning (Hall 2003). In these cases, twinning may be induced by disruption of communication between blastomeres at various stages of development (Fig. 1A-1, B-1 and C-1). With the improvement in microscopic and micromanipulator technology, mechanical induction of MZ twinning by bisection of pre-implantation embryos, generally morulae and blastocysts, has proven effective in a

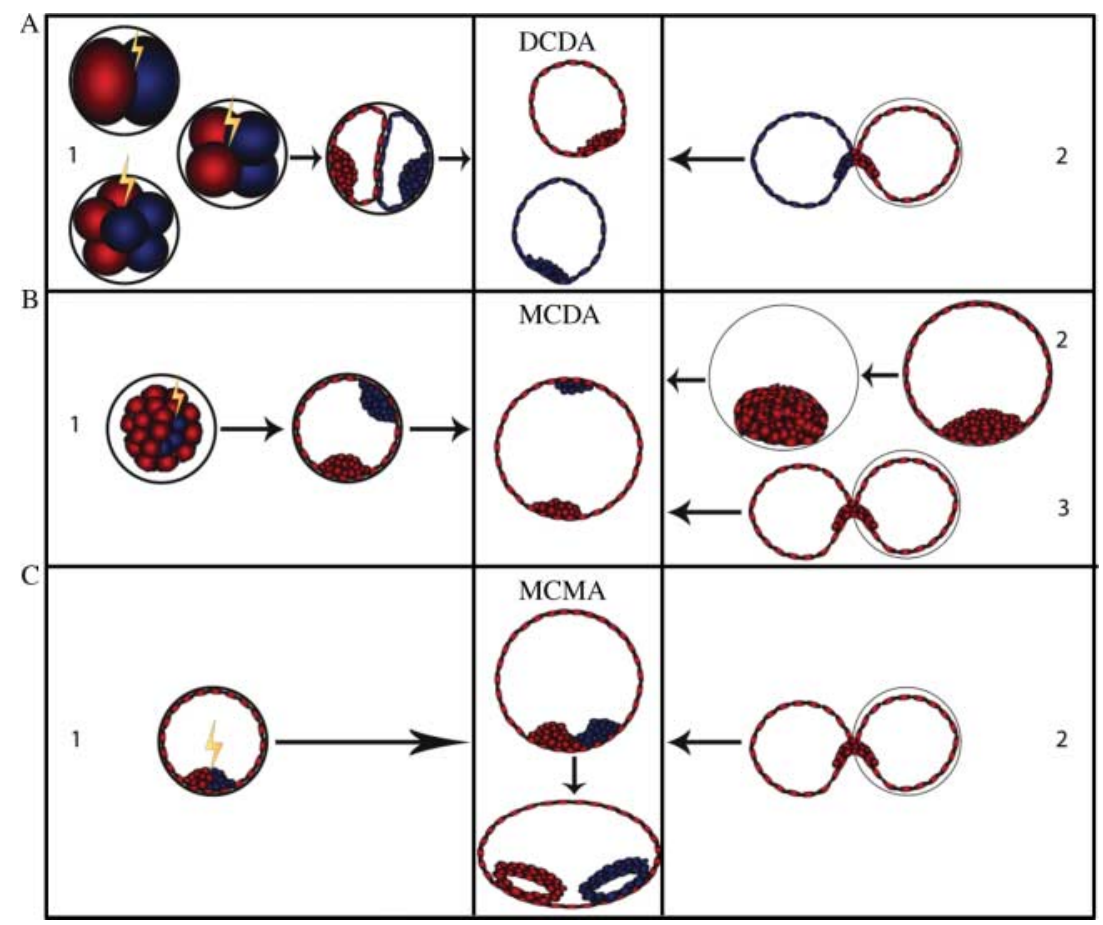

Figure 1 Potential mechanisms by which MZ twins might arise following ART. (A) DCDA MZ twins might result from: 1) insult to the early embryo by factors such as sub-optimal culture conditions or temperature fluctuations resulting in disruption of communication between blastomeres and the independent formation of two separate blastocysts. 2) Breach in the zona by ICSI or AH, or hardening of the zona by ovarian stimulation or suboptimal culture conditions resulting in abnormal blastocyst hatching. If hatching results in bisection of the trophectoderm and ICM, two separate blastocysts can form. (B) MCDA MZ twins might result from: 1) insult to the morula resulting in disruption of communication between inner blastomeres and the independent formation of two separate ICMs within the same blastocyst. 2) Blastocyst collapse, adhesion of ICM cells to another point within the trophectoderm, and the formation and growth of a second ICM. 3) Breach in or hardening of the zona resulting in abnormal blastocyst hatching. If hatching results in adhesion of ICM cells to another point within the trophectoderm, the formation and growth of a second ICM can result. (C) MCMA MZ twins might result from: 1) insult to the early blastocyst resulting in disruption of communication between early ICM blastomeres and the formation of two separate embryonic disks within the same blastocyst. 2) Breach in or hardening of the zona resulting in abnormal blastocyst hatching. If hatching results in disruption of communication between ICM cells (e.g. by apoptosis of some ICM cells), the formation of two separate embryonic disks within the same blastocyst can result. 
number of mammalian species, including cattle (Ozil et al. 1982), mice (Nagashima et al. 1984), sheep (Chesne et al. 1987), pigs (Reichelt \& Niemann 1994), and rhesus monkeys (Mitalipov et al. 2002).

Though MZ twinning occurs rarely if ever in mice (McLaren et al. 1995), it has been demonstrated that in vitro culture of mouse embryos can induce $\mathrm{MZ}$ twin formation in $\sim 1 \%$ of embryos cultured (Hsu \& Gonda 1980). The twinning was induced when hatched blastocysts attached to the culture dish antipolar to the ICM, and embryonic growth coupled with physical growth restrictions resulted in subdivision of the ICM. Another study reported the occurrence of double ICMs at a rate of 0.6 and $3.1 \%$ respectively for in vivo and in vitro fertilized mouse embryos prior to hatching (Chida 1990).

Two cases from human IVF suggest that double ICMs account for some $M Z$ pregnancies. One report exists on the day 5 transfer of two blastocysts, including one with a double ICM, resulting in a triplet pregnancy (Meintjes et al. 2001). Unexpectedly, the MZ twins were DCDA, indicating the trophectoderm was presumably split in the embryo. In another IVF laboratory, time-lapse photography of developing human IVF embryos documented the formation of two distinct ICMs in two of twenty-six embryos analyzed. The formation of double ICMs was believed to result from ectopic adhesion and subsequent proliferation of ICM cells on the opposing trophectoderm wall following blastocoel collapse and re-expansion (Fig. 1B-2; Payne et al. 2007).

The role of the zona on induction of $M Z$ twinning by mechanical splitting or ICM disruption of the hatching blastocyst has also been proposed (Edwards et al. 1986; Figs 1A-2, B-3, C-2 and 2). Factors such as thickening or hardening of the zona have been reported to contribute to twinning (Edwards et al. 1986, Tarlatzis et al. 2002).

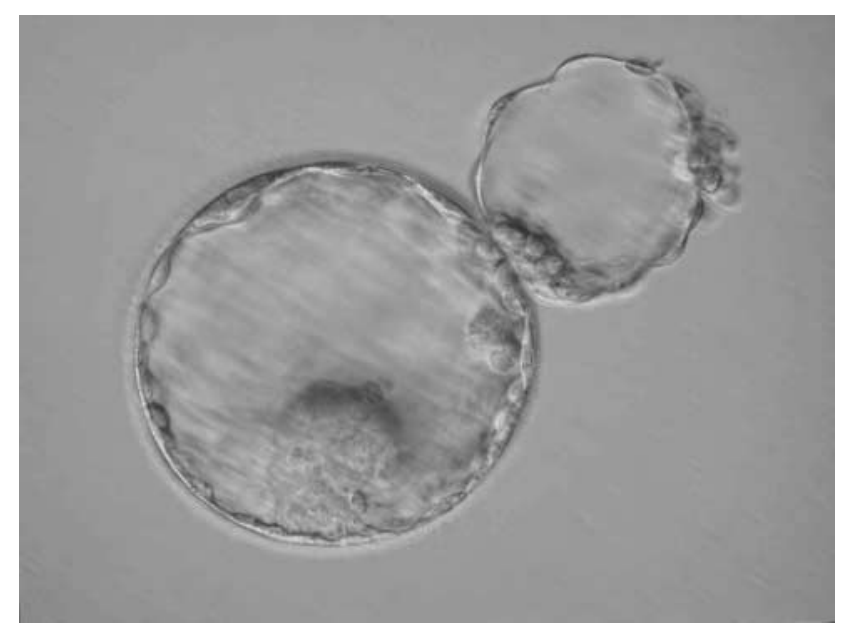

Figure 2 Abnormally hatching blastocyst following ICSI. Blastocyst hatching through a small hole in the zona as a result of zona hardening or zona manipulation may result in $\mathrm{MZ}$ twin formation by either complete embryo bisection or disruption of ICM cells.
While the zona may be involved in some cases of $M Z$ twinning, it is certainly not the exclusive mechanism as $\mathrm{MZ}$ twinning has been demonstrated to occur following transfer of zona-free blastocysts (Frankfurter et al. 2001).

Together, the multiple mechanisms of $M Z$ twinning observed and induced experimentally along with the occurrence of multiple placental arrangements in ART-associated $M Z$ pregnancies suggest that MZ twinning is likely associated with a variety of factors that may include delay in fertilization, embryo development, or implantation, mechanical disruption of the early embryo, and alterations in culture conditions. Indeed, a combination of factors is likely involved.

\section{Association of $\mathrm{MZ}$ twinning with ART}

Over two decades ago, it was observed that MZ twinning frequency was greater following ART procedures (Edwards et al. 1986) and artificial induction of ovulation (Derom et al. 1987). These early reports have been followed by numerous additional corroborating studies cited below; however, a large Danish cohort study recently published compared MZ and DZ twin rates between natural conceptions and conceptions following infertility treatment and found no association between fertility treatment and MZ twin deliveries (Zhu et al. 2007). Owing to the relatively limited number of individuals in the study undergoing infertility treatment and the small number of $M Z$ twins in the group, the authors were not able to analyze $M Z$ twinning rates based on treatment type. As a result, ICSI, IVF, intrauterine insemination, and hormone treatment cases were all analyzed together.

In the context of ART, there exists a great deal of debate over the factors that contribute to increased $M Z$ twinning as well as the mechanisms involved. Maternal age, ovarian stimulation, zona manipulation, temperature effects, and in vitro embryo culture have all been suggested as contributory factors in ART-associated MZ twinning. Table 1 summarizes reports investigating the impact of some of these factors on $\mathrm{MZ}$ twinning rates.

\section{Maternal age}

While there is some disagreement as to the impact of maternal age on MZ twinning rate (Steinman 2001), a $12-22 \%$ increase in $M Z$ twinning has been reported in women over the age of 35 compared with women younger than 25, while parity has been shown to have little or no effect (Bulmer 1970). On the other hand, an epidemiological review of a number of studies concluded that the rate of $M Z$ twinning is not affected by maternal age (Bortolus et al. 1999). More recent work in the context of IVF/ICSI treatment reported an increased risk of $\mathrm{MZ}$ twinning in women over the age of 35 (Abusheikha et al. 2000), although this conclusion was 
Table 1 Review of ART-associated MZ twinning publications.

\begin{tabular}{|c|c|c|c|c|c|}
\hline Reference & Type of ART & Total transfers & Total pregnancies & $\begin{array}{l}\text { Number of } M Z \\
\text { twin pregnancies }\end{array}$ & $\begin{array}{c}\text { MZ twin pregnancies/total } \\
\text { pregnancies }(\%)\end{array}$ \\
\hline \multicolumn{6}{|l|}{ Control } \\
\hline Derom et al. (1987) & None & & 99591 & 447 & 0.4 \\
\hline \multicolumn{6}{|l|}{ Ovulation induction } \\
\hline Derom et al. (1987) & $\mathrm{AOI}$ & & 1552 & 18 & 1.2 \\
\hline Kallen et al. (2002) & $\mathrm{AOI}$ & & 4029 & 31 & 0.8 \\
\hline Schachter et al. (2001) & $\mathrm{AOI}$ & & 129 & 2 & 1.6 \\
\hline \multicolumn{6}{|l|}{ Zona manipulation } \\
\hline \multirow[t]{2}{*}{ Alikani et al. (2003) } & IVF & & 2185 & 35 & 1.6 \\
\hline & ICSI & & 1537 & 37 & 2.4 \\
\hline \multirow[t]{2}{*}{ Elizur et al. (2004) } & IVF & & 389 & 4 & 1.0 \\
\hline & ICSI and/or $\mathrm{AH}$ & & 667 & 6 & 0.9 \\
\hline \multirow{2}{*}{ Hershlag et al. (1999) } & IVF & 559 & 141 & 0 & 0.0 \\
\hline & $\mathrm{AH}$ & 674 & 250 & 8 & 3.2 \\
\hline \multirow[t]{2}{*}{ Meldrum et al. (1998) } & IVF & & 320 & 3 & 0.9 \\
\hline & $\mathrm{AH}$ & & 136 & 1 & 0.7 \\
\hline \multirow[t]{2}{*}{ Milki et al. (2003) } & IVF & & 267 & 10 & 3.7 \\
\hline & ICSI and/or $\mathrm{AH}$ & & 287 & 8 & 2.8 \\
\hline \multirow[t]{2}{*}{ Saito et al. (2000) } & IVF & 3105 & 196 & 3 & 1.5 \\
\hline & ICSI, SUZI, or PZD & 1044 & 83 & 6 & 7.2 \\
\hline \multirow{2}{*}{ Schachter et al. (2001) } & IVF & 475 & 139 & 1 & 0.7 \\
\hline & ICSI and/or $\mathrm{AH}$ & 1438 & 463 & 4 & 0.9 \\
\hline \multirow{3}{*}{ Schieve et al. (2000) } & IVF & 21490 & 7163 & 9 & 0.1 \\
\hline & Mix IVF $+\mathrm{AH}$ & 3310 & 1327 & 2 & 0.2 \\
\hline & $\mathrm{AH}$ & 10703 & 3650 & 12 & 0.3 \\
\hline \multirow{4}{*}{ Sills et al. (2000b) } & IVF & & 121 & 3 & 2.5 \\
\hline & ICSI & & 151 & 1 & 0.7 \\
\hline & $\mathrm{AH}$ & & 589 & 8 & 1.4 \\
\hline & $\mathrm{ICSI}$ and $\mathrm{AH}$ & & 813 & 7 & 0.9 \\
\hline \multirow[t]{2}{*}{ Tarlatzis et al. (2002) } & IVF & 48 & 15 & 0 & 0.0 \\
\hline & ICSI & 79 & 33 & 6 & 18.2 \\
\hline \multicolumn{6}{|l|}{ Embryo freezing } \\
\hline \multirow[t]{2}{*}{ Alikani et al. (2003) } & Fresh ET & & 3250 & 72 & 2.2 \\
\hline & Frozen-thawed ET & & 511 & 9 & 1.8 \\
\hline \multicolumn{6}{|l|}{ Blastocyst transfer } \\
\hline Behr et al. (2000) & D5 transfer & & 199 & 10 & 5.0 \\
\hline \multirow{2}{*}{ da Costa et al. (2001) } & D3 transfer & 2584 & 814 & 6 & 0.7 \\
\hline & D5 transfer & 335 & 186 & 5 & 2.7 \\
\hline \multirow[t]{2}{*}{ Jain et al. (2004) } & D3 transfer & 90 & 47 & 1 & 2.1 \\
\hline & D5 transfer & 75 & 38 & 5 & 13.2 \\
\hline Milki et al. (2003) & D3 transfer & & 357 & 7 & 2.0 \\
\hline & D5 transfer & & 197 & 11 & 5.6 \\
\hline Moayeri et al. (2007) & D3 transfer & & 547 & 10 & 1.8 \\
\hline & D5 transfer & & 385 & 9 & 2.3 \\
\hline Papanikolaou et al. (2006) & D2 transfer & 176 & 41 & 2 & 4.9 \\
\hline & D5 transfer & 175 & 58 & 0 & 0.0 \\
\hline Rijnders et al. (1998) & D2 transfer & & 960 & 5 & 0.5 \\
\hline & D3 transfer & & 735 & 5 & 0.7 \\
\hline & D5 transfer & & 111 & 3 & 2.7 \\
\hline Sheiner et al. (2001) & D3 transfer & & 204 & 3 & 1.5 \\
\hline & D5 transfer & & 9 & 2 & 22.2 \\
\hline Wright et al. (2004) & D2 transfer & 6679 & 2337 & 5 & 0.2 \\
\hline & D3 transfer & 77256 & 29144 & 117 & 0.4 \\
\hline & D4 transfer & 3169 & 1010 & 4 & 0.4 \\
\hline & D5 transfer & 17628 & 7921 & 119 & 1.5 \\
\hline & D6 transfer & 3604 & 1122 & 10 & 0.9 \\
\hline
\end{tabular}

$\mathrm{AOI}$, artificial ovulation induction; $\mathrm{AH}$, assisted hatching; $\mathrm{ART}$, assisted reproductive technologies; $\mathrm{D}$, day; ICSI, intracytoplasmic sperm injection; IVF, conventional in vitro fertilization; MZ, monozygotic; PZD, partial zona dissection; SUZI, sub-zonal insemination.

based on trends rather than statistical significance, and the numbers of $M Z$ twins in the study were small. Likewise, Alikani et al. (2003) reported a trend $(P=0.07)$ toward increasing $\mathrm{MZ}$ twin rates following IVF associated with advanced maternal age, while a more recent report that evaluated monochorionic MZ twins exclusively found no association between maternal age and monochorionic twin occurrence (Skiadas et al. 2008). These limited data indicate that increasing maternal age may contribute to an increased incidence of $M Z$ twins, but the contribution is likely minimal in ART cases. 


\section{Ovarian stimulation}

The earliest report of increased MZ twinning following ovarian stimulation was based on data collected from the East Flanders Prospective Twin Study (EFPTS), an ongoing population-based study of the East Flanders Province in Belgium, which was initiated in 1964. Evaluation of zygosity of 2648 multiple births indicated an $M Z$ twinning rate of $1.2 \%$ following ovarian stimulation, approximately three times higher than the incidence in spontaneously conceived pregnancies (Derom et al. 1987). More recently, Kallen et al. (2002) indicated that women undergoing ovarian stimulation delivered MZ twins at nearly twice the rate of controls. A later EFPTS analysis indicated that a higher proportion of twins conceived following ovarian stimulation with clomiphene citrate were MZ compared with iatrogenic cases where no clomiphene citrate was used (Derom et al. 2006). Another study concluded MZ frequency was more closely associated with controlled ovarian stimulation and ovulation induction by gonadotropin therapy than other ART procedures with an overall $M Z$ rate of $0.95 \%$ for all ART procedures and a rate of $1.5 \%$ following ovulation induction or ovarian stimulation alone (Schachter et al. 2001). It has been suggested that this increased incidence of $M Z$ twins may be a result of either a delay in implantation (Van der Auwera \& D'Hooghe 2001, Steinman 2001) or hardening of the zona (Longo 1981, Edwards et al. 1986, Derom et al. 1987 ) induced by ovulatory hormones.

\section{Zona pellucida manipulation}

Two primary zona manipulation techniques are commonly utilized in assisted reproduction to improve fertilization and implantation rates. ICSI involves direct injection of a spermatozoon into the oocyte cytoplasm. The technique greatly reduces the number of sperm needed for IVF and allows the use of sub- or non-motile sperm. The first ICSI pregnancy was achieved in 1991 (Palermo et al. 1992). Since that time, ICSI has become a common procedure for some forms of male factor infertility.

The other zona manipulation technique, assisted hatching, involves chemical, mechanical, or laser manipulation of the zona pellucida of a fertilized embryo in an effort to facilitate embryo hatching and enhance the probability of implantation. Since the initial report in 1990 demonstrating its effectiveness in improving implantation in some patients (Cohen et al. 1990), AH has also become a common practice in ART.

It has been proposed that an artificial breach in the zona by ICSI or $\mathrm{AH}$ and subsequent herniation of blastomeres through the hole in the zona during blastocyst expansion might result in mechanical splitting of the blastocyst and formation of MZ twins (Talansky \& Gordon 1988). This phenomenon has been recorded by time-lapse photography in bovine embryos (Van Langendonckt et al. 2000).

There are a number of reports indicating zona manipulation increases the risk for $M Z$ twinning (Hershlag et al. 1999, Abusheikha et al. 2000, Saito et al. 2000, Schieve et al. 2000, Tarlatzis et al. 2002, Skiadas et al. 2008); conversely, there are several studies that demonstrate no association between zona manipulation and increased MZ twinning rates (Meldrum et al. 1998, Behr et al. 2000, Sills et al. 2000b, Schachter et al. 2001, Milki et al. 2003, Elizur et al. 2004, Yanaihara et al. 2007).

An early report of six cases of $M Z$ twins observed that each of the six cases was associated either with naturally thin zonas or with some sort of zona manipulation. In the same retrospective study, no $M Z$ twins resulted from transfers of embryos with normal zona thickness or embryos with intact zonas (Alikani et al. 1994). Hershlag et al. reported no MZ twins occurred out of 559 unhatched embryo transfers and one $M Z$ triplet and seven $M Z$ twin pregnancies resulted from 674 hatched embryo transfers $(1.2 \% ; P<0.01)$. However, the unhatched embryos were transferred between 1990 and 1993, and the hatched embryos were transferred between 1994 and 1996, and there is no indication of the stages of embryos transferred (Hershlag et al. 1999).

Analysis of data collected through a national ART survey in Japan between 1994 and 1995 representing nine $M Z$ twin pregnancies out of 279 (3.2\%) total pregnancies also revealed a trend toward increased incidence of $\mathrm{MZ}$ twins following zona manipulation techniques with an apparent increased risk associated with larger breaches in the zona (Saito et al. 2000). In another case, six MZ pregnancies resulted from 102 cycles following ICSI (5.9\%), and no MZ pregnancies were detected in 79 cycles following conventional IVF $(P=0.033$; Tarlatzis et al. 2002). Retrospective analysis by Alikani et al. (2003) of 81 cycles resulting in MZ twins in one clinic indicated an association between zona manipulation and $\mathrm{MZ}$ twinning with $2.4 \%$ of $\mathrm{MZ}$ twins occurring following ICSI cycles and $1.6 \%$ following standard insemination, but the difference was not significant. Two other reports also suggest a possible correlation between zona manipulation and $\mathrm{MZ}$ twinning rate (Abusheikha et al. 2000, Schieve et al. 2000). A recent study evaluated risk factors specifically for monochorionic twinning and found a significant association between ICSI and monochorionic twins as well as an association approaching significance between $\mathrm{AH}$ and monochorionic twins (Skiadas et al. 2008).

A major limitation of studies evaluating the effect of zona manipulation on $M Z$ twinning rate is the extremely low incidence of $M Z$ twinning even following ART procedures. With that limitation in mind, it is certainly easier to demonstrate a lack of significance than to show significance; however, it is clear that additional studies with greater statistical power will be necessary in order 
to more fully address the question of whether zona manipulation results in an overall increased incidence of MZ twinning.

\section{Temperature effects}

As it has been demonstrated that temperature fluctuations in developing fish embryos result in increased $M Z$ twinning (Stockard 1921), it has been proposed that there may be a temperature effect in ART-associated MZ twinning. The only association with temperature changes and $M Z$ twinning that have been addressed is the incidence of $M Z$ twinning following the transfer of frozen-thawed embryos. There has been brief mention of the potential for such an association (Toledo 2005, Faraj et al. 2007), but other reports indicate no relationship between the transfer of frozen-thawed embryos and $M Z$ twinning rate (Alikani et al. 2003, Blickstein et al. 2003). If embryo cryopreservation results in increased $M Z$ twinning, the increase is likely associated with zona hardening rather than a direct temperature effect. The involvement of minor temperature fluctuations on $M Z$ twinning in manipulated and in vitro cultured embryos has not been addressed but may warrant investigation as embryos undergoing these procedures are subject to temperature changes when removed from the incubator for any period of time.

\section{Blastocyst transfer}

The first report of $M Z$ twins resulting from the day 5 blastocyst transfer without zona manipulation was in 1999 when one case of two-blastocyst transfer resulted in an MCDA set of twins and another resulted in a singleton and an MCMA set of twins (Peramo et al. 1999). Blastocyst embryo transfer generally results in improved pregnancy rates compared with cleavagestage embryo transfers (Wilson et al. 2002, Papanikolaou et al. 2006); however, a number of reports have suggested that blastocyst transfer may result in increased $M Z$ twinning rate.

An early retrospective study by Behr et al. (2000) collected data from multiple IVF laboratories to analyze MZ pregnancy rates following blastocyst transfer. In all, ten $M Z$ twin pregnancies resulted, yielding an overall incidence of $5.0 \%$. Several other smaller studies have reported increased MZ twinning associated with blastocyst transfer (Rijnders et al. 1998, Sheiner et al. 2001). Analysis of data reported by $93 \%$ of all US ART laboratories to the Centers for Disease Control and Prevention for embryo transfer procedures performed in 1999 and 2000 also demonstrated a link between blastocyst transfer and increased MZ twinning rates. Pregnancies for which the number of ultrasounddetectable fetal heartbeats exceeded the number of transferred embryos were considered to contain at least one set of MZ twins. Out of 39198 pregnancies, $226 \mathrm{MZ}$ twins were detected $(0.6 \%)$. A significantly greater proportion of $M Z$ pregnancies were derived from the day 5 or 6 embryo transfers compared with the cleavagestage transfers (Wright et al. 2004).

Another retrospective study of ICSI embryos found MZ twin occurrence in 5/129 (3.9\%) blastocyst transfer pregnancies occurring between 1998 and 1999 compared with only $6 / 814(0.7 \%)$ following four- to eight-cell transfers performed from 1996 to 1999 $(P<0.001$; da Costa et al. 2001). Similarly Jain et al. compared the $M Z$ twinning rate of 96 day 3 embryo transfers performed between 1997 and 1998 with 75 blastocyst transfers occurring between 1999 and 2000 . They found that $1 / 47(2.1 \%)$ day 3 embryo transfer pregnancies were MZ twins compared with 5/32 (13.2\%) following blastocyst transfer (Jain et al. 2004). A somewhat larger retrospective study by Milki et al. (2003) analyzing pregnancy data generated concurrently from cleavage-stage and blastocyst transfers also reported significantly higher incidence of $M Z$ twins following blastocyst transfer $(5.6 \%)$ compared with cleavage-stage embryo transfer $(2.0 \% ; P<0.03)$. Four years later, this same group performed a similar analysis to determine whether increased experience with blastocyst transfer impacted $M Z$ twinning rates. Results of the more recent study indicated that $M Z$ twinning rates associated with blastocyst transfer had declined over time so that recent $M Z$ twinning rates following blastocyst transfer $(2.3 \%)$ were not significantly different from rates in the cleavage-stage embryo transfer group (1.8\%; Moayeri et al. 2007). Their conclusion was that improved culture conditions as well as increased experience with blastocyst embryo culture and transfer contributed to the decline in MZ twinning. The recent study by Skiadas et al. (2008) that reported an association between zona manipulation and the incidence of monochorionic twins also found a significantly higher incidence of monochorionic twins following day 5 embryo transfer. Further analysis by the same group found that the combination of zona manipulation and day 5 embryo transfer had a compounding effect on monochorionic twinning rates as an increased incidence of monochorionic twins occurred following day 5 transfer of ICSI embryos compared with either day 3 transfer of ICSI embryos or day 5 transfer of non-ICSI embryos (Skiadas et al. 2008). The phenomenon of a synergistic effect of multiple factors on $M Z$ twinning rates had not been demonstrated in previous studies.

A recent randomized trial of women under the age of 36 undergoing single embryo transfer with day 3 or day 5 embryos found significantly higher clinical pregnancy and delivery rates following blastocyst transfer. In this group, there were no MZ pregnancies out of 73 pregnancies derived from blastocyst transfer, and there were two $M Z$ twin pregnancies out of 59 day 3 embryo transfer pregnancies (3.4\%; Papanikolaou et al. 2006). 
As is the case with reports linking zona manipulation with $M Z$ twinning, there is not complete consensus that blastocyst transfer inherently results in increased $M Z$ twinning rates. However, the majority of reports, particularly larger studies, seem to suggest an association between blastocyst transfer and MZ twinning rates. Based on available data, it seems likely that blastocyst embryo transfer contributes to the increased incidence of $\mathrm{MZ}$ twinning, but it is certainly not the sole factor.

\section{Sub-optimal culture conditions}

Hardening of the zona following prolonged embryo culture (Behr et al. 2000) or sub-optimal culture conditions leading to splitting of the ICM by selective apoptosis (Menezo \& Sakkas 2002) or blastocoel collapse (Payne et al. 2007) in embryos grown in vitro have been proposed as mechanisms contributing to increased $M Z$ twin rates in blastocyst transfer pregnancies. Interestingly, two groups have noted the absence of $\mathrm{MZ}$ twins following embryo transfer of blastocysts grown with co-culture (Behr et al. 2000, Menezo \& Sakkas 2002), lending credence to the contribution of extended culture under sub-optimal conditions to the increased incidence of $M Z$ twinning. Menezo's group later reported the effect of prolonged embryo culture on $M Z$ twinning rates was dependent on the culture media, suggesting that improved culture media may reduce the incidence of $\mathrm{MZ}$ twins following IVF (Cassuto et al. 2003). In addition, the two studies by Milki et al. discussed previously suggest that increased experience in embryo culture or improved embryo culture conditions resulted in decreased rates of $M Z$ twinning (Milki et al. 2003, Moayeri et al. 2007).

\section{Conclusions}

There are several factors that confound the characterization of elements contributing to ART-associated $M Z$ twinning. A primary limitation is the lack of a basic understanding of the mechanisms involved in spontaneous $M Z$ twinning. In addition, the extremely low occurrence of spontaneous and ART-associated MZ twins greatly increases the number of study cases necessary to establish appropriate statistical power. By necessity, studies evaluating $M Z$ twin occurrence are retrospective, and the majority contain mixed treatments and/or potentially confounding variables, which further lessen their statistical power. Monozygosity can be diagnosed by ultrasound based on membrane arrangement or post partum based on membrane arrangement, physical similarity or genetic testing, the latter being the most reliable. Following IVF, in many cases, more than one embryo is transferred so only monochorionic twins can be diagnosed by ultrasound to be $M Z$ thus missing DCDA MZ twins, which in spontaneous $M Z$ twins can account for a quarter or more of the cases (Hall 2003).
Additionally, postpartum assessment of zygosity following multiple embryo transfer fails to include vanishing twins or complete miscarriages that have been reported to occur in 12.1 and $5.0 \%$ of IVF/ICSI twin pregnancies respectively. In light of these facts, the true incidence of $M Z$ twins is likely greater than the reported incidence in many cases.

Routine determination of both zygosity and chorionicity of all multiple births will greatly aid in understanding the timing and associated mechanisms involved in $M Z$ twinning. The Society for Assisted Reproductive Technologies, ESHRE and other reporting agencies could add this to the information collected from reporting clinics. Further, the increasing use of single embryo transfer will shed light on the true incidence of $M Z$ twinning.

While the data regarding chorionicity are too sparse to determine the overall occurrence of each type of placentation, the fact that all three configurations of MZ placentation have been observed following ART indicates that $M Z$ twinning occurs at various times during embryo development and by a variety of mechanisms.

Based on the reports reviewed here, it is evident that a number of factors likely contribute to increased $M Z$ twinning associated with ART; however, some factors are probably more important than others. Among the larger studies, the factors that seem to make the most significant contributions to $M Z$ twinning are ovarian stimulation and extended embryo culture coupled with blastocyst transfer. Additional, larger, and more detailed studies will allow for better assessment of the relative risks of each of the potentially contributory factors to $M Z$ twinning. By increasing our understanding of the incidence as well as the causal factors of $M Z$ twinning in ART, we will be better equipped to reduce its incidence and thus avoid the associated complications to fetus and mother.

\section{Declaration of interest}

The authors declare no conflict of interest associated with the content of this review.

\section{Funding}

This review was prepared without financial support.

\section{References}

Abusheikha N, Salha O, Sharma V \& Brinsden P 2000 Monozygotic twinning and ivf/icsi treatment: a report of 11 cases and review of literature. Human Reproduction Update 6 396-403.

Alikani M, Noyes N, Cohen J \& Rosenwaks Z 1994 Monozygotic twinning in the human is associated with the zona pellucida architecture. Human Reproduction 9 1318-1321.

Alikani M, Cekleniak NA, Walters E \& Cohen J 2003 Monozygotic twinning following assisted conception: an analysis of 81 consecutive cases. Human Reproduction 18 1937-1943. 
Andersen AN, Goossens V, Gianaroli L, Felberbaum R, de Mouzon J \& Nygren KG 2007 Assisted reproductive technology in Europe, 2003. Results generated from European registers by ESHRE. Human Reproduction 22 1513-1525.

Van der Auwera I \& D'Hooghe T 2001 Superovulation of female mice delays embryonic and fetal development. Human Reproduction $\mathbf{1 6}$ 1237-1243.

Beemsterboer SN, Homburg R, Gorter NA, Schats R, Hompes PG \& Lambalk CB 2006 The paradox of declining fertility but increasing twinning rates with advancing maternal age. Human Reproduction $\mathbf{2 1}$ 1531-1532.

Behr B, Fisch JD, Racowsky C, Miller K, Pool TB \& Milki AA 2000 Blastocyst-et and monozygotic twinning. Journal of Assisted Reproduction and Genetics 17 349-351.

Blickstein I 2005 Estimation of iatrogenic monozygotic twinning rate following assisted reproduction: pitfalls and caveats. American Journal of Obstetrics and Gynecology 192 365-368.

Blickstein I \& Keith LG 2007 On the possible cause of monozygotic twinning: lessons from the 9-banded armadillo and from assisted reproduction. Twin Research and Human Genetics 10 394-399.

Blickstein I, Verhoeven HC \& Keith LG 1999 Zygotic splitting after assisted reproduction. New England Journal of Medicine 340 738-739.

Blickstein I, Jones C \& Keith LG 2003 Zygotic-splitting rates after singleembryo transfers in in vitro fertilization. New England Journal of Medicine 348 2366-2367.

Bortolus R, Parazzini F, Chatenoud L, Benzi G, Bianchi MM \& Marini A 1999 The epidemiology of multiple births. Human Reproduction Update 5 179-187.

Bulmer MG 1970 The Biology of Twinning in Man. Oxford, UK: Clarendon Press.

Cassuto G, Chavrier M \& Menezo Y 2003 Culture conditions and not prolonged culture time are responsible for monozygotic twinning in human in vitro fertilization. Fertility and Sterility $\mathbf{8 0} 462-463$.

Chesne P, Colas G, Cognie Y, Guerin Y \& Sevellec C 1987 Lamb production using superovulation, embryo bisection, and transfer. Theriogenology 27 751-757.

Chida S 1990 Monozygous double inner cell masses in mouse blastocysts following fertilization in vitro and in vivo. Journal of In Vitro Fertilization and Embryo Transfer 7 177-179.

Cohen J, Elsner C, Kort H, Malter H, Massey J, Mayer MP \& Wiemer K 1990 Impairment of the hatching process following ivf in the human and improvement of implantation by assisting hatching using micromanipulation. Human Reproduction 5 7-13.

da Costa Al, Abdelmassih S, de Oliveira FG, Abdelmassih V, Abdelmassih R, Nagy ZP \& Balmaceda JP 2001 Monozygotic twins and transfer at the blastocyst stage after icsi. Human Reproduction 16 333-336.

Derom C, Vlietinck R, Derom R, Van den Berghe H \& Thiery M 1987 Increased monozygotic twinning rate after ovulation induction. Lancet $\mathbf{1}$ 1236-1238.

Derom C, Leroy F, Vlietinck R, Fryns JP \& Derom R 2006 High frequency of iatrogenic monozygotic twins with administration of clomiphene citrate and a change in chorionicity. Fertility and Sterility 85 755-757.

Dickinson JE 2005 Monoamniotic twin pregnancy: a review of contemporary practice. Australian and New Zealand Journal of Obstetrics and Gynaecology 45 474-478.

Edwards RG, Mettler L \& Walters DE 1986 Identical twins and in vitro fertilization. Journal of In Vitro Fertilization and Embryo Transfer 3 114-117.

Elizur SE, Levron J, Shrim A, Sivan E, Dor J \& Shulman A 2004 Monozygotic twinning is not associated with zona pellucida micromanipulation procedures but increases with high-order multiple pregnancies. Fertility and Sterility 82 500-501.

Enders AC 1962 The structure of the armadillo blastocyst. Journal of Anatomy 96 39-48.

Enders AC 2002a Implantation in the nine-banded armadillo: how does a single blastocyst form four embryos? Placenta 23 71-85.

Enders AC 2002 $b$ Formation of monozygotic twins: when does it occur? Placenta 23 236-238.

Faraj R, Evbuomwan I, Sturgiss S \& Aird I 2008 Monozygotic triplet pregnancy following egg donation and transfer of single frozen-thawed embryo. Fertility and Sterility 89 1260.e9-1260.e12.
Fieni S, Gramellini D, Piantelli G, Verrotti C \& Cavallotti D 2004 Twin-twin transfusion syndrome: a review of treatment option. Acta Bio-Medica 75 34-39.

Frankfurter D, Hackett R, Meng L \& Keefe DL 2001 Complete removal of the zona pellucida by pronase digestion prior to blastocyst embryo transfer does not eliminate monozygotic pregnancies following IVF. Fertility and Sterility 76 S144.

Ghulmiyyah LM, Perloe M, Tucker MJ, Zimmermann JH, Eller DP \& Sills ES 2003 Monochorionic-triamniotic triplet pregnancy after intracytoplasmic sperm injection, assisted hatching, and two-embryo transfer: first reported case following ivf. BMC Pregnancy and Childbirth 34.

Hack KE, Derks JB, de Visser VL, Elias SG \& Visser GH 2006 The natural course of monochorionic and dichorionic twin pregnancies: a historical cohort. Twin Research and Human Genetics 9 450-455.

Hall JG 2003 Twinning. Lancet 362 735-743.

Hamamy HA, Ajlouni HK \& Ajlouni KM 2004 Familial monozygotic twinning: report of an extended multi-generation family. Twin Research 7 219-222.

Harkness UF \& Crombleholme TM 2005 Twin-twin transfusion syndrome: where do we go from here? Seminars in Perinatology 29 296-304.

Henne MB, Milki AA \& Westphal LM 2005 Monochorionic triplet gestation after in vitro fertilization using donor oocytes: case report and review. Fertility and Sterility 83 742-748.

Hershlag A, Paine T, Cooper GW, Scholl GM, Rawlinson K \& Kvapil G 1999 Monozygotic twinning associated with mechanical assisted hatching. Fertility and Sterility 71 144-146.

Hsu YC \& Gonda MA 1980 Monozygotic twin formation in mouse embryos in vitro. Science 209 605-606.

Jain JK, Boostanfar R, Slater CC, Francis MM \& Paulson RJ 2004 Monozygotic twins and triplets in association with blastocyst transfer. Journal of Assisted Reproduction and Genetics 21 103-107.

Kallen B, Olausson PO \& Nygren KG 2002 Neonatal outcome in pregnancies from ovarian stimulation. Obstetrics and Gynecology 100 414-419.

Van Langendonckt A, Wyns C, Godin PA, Toussaint-Demylle D \& Donnez J 2000 Atypical hatching of a human blastocyst leading to monozygotic twinning: a case report. Fertility and Sterility 74 1047-1050.

Longo FJ 1981 Changes in the zones pellucidae and plasmalemma of aging mouse eggs. Biology of Reproduction 25 399-411.

MacGillivray I 1986 Epidemiology of twin pregnancy. Seminars in Perinatology 10 4-8.

McLaren A, Molland P \& Signer E 1995 Does monozygotic twinning occur in mice? Genetical Research 66 195-202.

Meintjes M, Guerami AR, Rodriguez JA, Crider-Pirkle SS \& Madden JD 2001 Prospective identification of an in vitro-assisted monozygotic pregnancy based on a double-inner-cell-mass blastocyst. Fertility and Sterility 76 S172-S173.

Meldrum DR, Wisot A, Yee B, Garzo G, Yeo L \& Hamilton F 1998 Assisted hatching reduces the age-related decline in ivf outcome in women younger than age 43 without increasing miscarriage or monozygotic twinning. Journal of Assisted Reproduction and Genetics 15 418-421.

Menezo YJ \& Sakkas D 2002 Monozygotic twinning: is it related to apoptosis in the embryo? Human Reproduction 17 247-248.

Milki AA, Jun SH, Hinckley MD, Behr B, Giudice LC \& Westphal LM 2003 Incidence of monozygotic twinning with blastocyst transfer compared to cleavage-stage transfer. Fertility and Sterility 79 503-506.

Mitalipov SM, Yeoman RR, Kuo HC \& Wolf DP 2002 Monozygotic twinning in rhesus monkeys by manipulation of in vitro-derived embryos. Biology of Reproduction 66 1449-1455.

Moayeri SE, Behr B, Lathi RB, Westphal LM \& Milki AA 2007 Risk of monozygotic twinning with blastocyst transfer decreases over time: an 8-year experience. Fertility and Sterility 87 1028-1032.

Nagashima H, Matsui K, Sawasaki T \& Kano Y 1984 Production of monozygotic mouse twins from microsurgically bisected morulae. Journal of Reproduction and Fertility 70 357-362.

Ozil JP, Heyman Y \& Renard JP 1982 Production of monozygotic twins by micromanipulation and cervical transfer in the cow. Veterinary Record 110 126-127.

Palermo G, Joris H, Devroey P \& Van Steirteghem AC 1992 Pregnancies after intracytoplasmic injection of single spermatozoon into an oocyte. Lancet 340 17-18. 
Papanikolaou EG, Camus M, Kolibianakis EM, Van Landuyt L, Van Steirteghem A \& Devroey P 2006 In vitro fertilization with single blastocyst-stage versus single cleavage-stage embryos. New England Journal of Medicine 354 1139-1146.

Parisi P, Gatti M, Prinzi G \& Caperna G 1983 Familial incidence of twinning. Nature 304 626-628.

Payne D, Okuda A, Wakatsuki Y, Takeshita C, Iwata K, Shimura T, Yumoto K, Ueno Y, Flaherty S \& Mio Y 2007 Time-lapse recording identifies human blastocysts at risk of producing monozygotic twins. Human Reproduction 22 (Supplement 1) i9-i10.

Peramo B, Ricciarelli E, Cuadros-Fernandez JM, Huguet E \& Hernandez ER 1999 Blastocyst transfer and monozygotic twinning. Fertility and Sterility 72 1116-1117.

Putterman S, Figueroa R, Garry D \& Maulik D 2003 Comparison of obstetric outcomes in twin pregnancies after in vitro fertilization, ovarian stimulation and spontaneous conception. Journal of Maternal-Fetal and Neonatal Medicine 14 237-240.

Rao TV 1978 Maternal age, parity, and twin pregnancies. Progress in Clinical and Biological Research 24 99-103.

Rao A, Sairam S \& Shehata H 2004 Obstetric complications of twin pregnancies. Best Practice \& Research. Clinical Obstetrics \& Gynaecology $18557-576$.

Reichelt B \& Niemann H 1994 Generation of identical twin piglets following bisection of embryos at the morula and blastocyst stage. Journal of Reproduction and Fertility 100 163-172.

Rijnders PM, van Os HC \& Jansen CAM 1998 Increased incidence of monozygotic twinning following the transfer of blastocysts in human IVF/ICSI. Fertility and Sterility 70 S16-S17.

Risquez F, Gil M, D'Ommar G, Poo M, Sosa A \& Piras M 2004 Monochorionic triplets after single embryo transfer. Reproductive Biomedicine Online 9 370-371.

Saito H, Tsutsumi O, Noda Y, Ibuki Y \& Hiroi M 2000 Do assisted reproductive technologies have effects on the demography of monozygotic twinning? Fertility and Sterility 74 178-179.

Schachter M, Raziel A, Friedler S, Strassburger D, Bern O \& Ron-El R 2001 Monozygotic twinning after assisted reproductive techniques: a phenomenon independent of micromanipulation. Human Reproduction 16 1264-1269.

Schieve LA, Meikle SF, Peterson HB, Jeng G, Burnett NM \& Wilcox LS 2000 Does assisted hatching pose a risk for monozygotic twinning in pregnancies conceived through in vitro fertilization? Fertility and Sterility $\mathbf{7 4}$ 288-294.

Scott L 2002 The origin of monozygotic twinning. Reproductive Biomedicine Online 5 276-284.

Sheiner E, Har-Vardi I \& Potashnik G 2001 The potential association between blastocyst transfer and monozygotic twinning. Fertility and Sterility 75 217-218.

Sills ES, Tucker MJ \& Palermo GD 2000a Assisted reproductive technologies and monozygous twins: implications for future study and clinical practice. Twin Research 3 217-223.

Sills ES, Moomjy M, Zaninovic N, Veeck LL, McGee M, Palermo GD \& Rosenwaks Z 2000b Human zona pellucida micromanipulation and monozygotic twinning frequency after IVF. Human Reproduction 15 890-895.

Skiadas CC, Missmer SA, Benson CB, Gee RE \& Racowsky C 2008 Risk factors associated with pregnancies containing a monochorionic pair following assisted reproductive technologies. Human Reproduction 23 1366-1371.
Souter VL, Kapur RP, Nyholt DR, Skogerboe K, Myerson D, Ton CC, Opheim KE, Easterling TR, Shields LE, Montgomery GW et al. 2003 A report of dizygous monochorionic twins. New England Journal of Medicine 349 154-158.

Steinman G 2001 Mechanisms of twinning. II. Laterality and intercellular bonding in monozygotic twinning. Journal of Reproductive Medicine $\mathbf{4 6}$ 473-479.

Steinman G 2003 Mechanisms of twinning. VI. Genetics and the etiology of monozygotic twinning in in vitro fertilization. Journal of Reproductive Medicine 48 583-590.

Stockard CR 1921 Developmental rate and structural expression: an experimental study of twins, double monsters and single deformities, and the interaction among embryonic organs during their origin and development. American Journal of Anatomy 28 115-262.

Talansky BE \& Gordon JW 1988 Cleavage characteristics of mouse embryos inseminated and cultured after zona pellucida drilling. Gamete Research 21 277-287.

Tarlatzis BC, Qublan HS, Sanopoulou T, Zepiridis L, Grimbizis G \& Bontis J 2002 Increase in the monozygotic twinning rate after intracytoplasmic sperm injection and blastocyst stage embryo transfer. Fertility and Sterility 77 196-198.

Toledo MG 2005 Is there increased monozygotic twinning after assisted reproductive technology? Australian and New Zealand Journal of Obstetrics and Gynaecology 45 360-364.

Unger S, Hoopmann M, Bald R, Foth D \& Nawroth F 2004 Monozygotic triplets and monozygotic twins after ICSI and transfer of two blastocysts: case report. Human Reproduction 19 110-113.

Wenstrom KD, Syrop CH, Hammitt DG \& Van Voorhis BJ 1993 Increased risk of monochorionic twinning associated with assisted reproduction. Fertility and Sterility 60 510-514.

White C \& Wyshak G 1964 Inheritance in human dizygotic twinning. New England Journal of Medicine 271 1003-1005.

Wilson M, Hartke K, Kiehl M, Rodgers J, Brabec C \& Lyles R 2002 Integration of blastocyst transfer for all patients. Fertility and Sterility $\mathbf{7 7}$ 693-696.

Wright V, Schieve LA, Vahratian A \& Reynolds MA 2004 Monozygotic twinning associated with day 5 embryo transfer in pregnancies conceived after IVF. Human Reproduction 19 1831-1836.

Wright VC, Chang J, Jeng G, Chen M \& Macaluso M 2007 Assisted reproductive technology surveillance - United States, 2004. Morbidity and Mortality Weekly Report. Surveillance Summaries 56 1-22.

Yanaihara A, Yorimitsu T, Motoyama H, Watanabe H \& Kawamura T 2007 Monozygotic multiple gestation following in vitro fertilization: analysis of seven cases from japan. Journal of Experimental \& Clinical Assisted Reproduction 44.

Yovich JL, Stanger JD, Grauaug A, Barter RA, Lunay G, Dawkins RL \& Mulcahy MT 1984 Monozygotic twins from in vitro fertilization. Fertility and Sterility 41 833-837.

Zhu JL, Basso O, Obel C, Christensen K \& Olsen J 2007 Infertility, infertility treatment and twinning: the danish national birth cohort. Human Reproduction 22 1086-1090.

Received 14 May 2008

First decision 13 June 2008

Accepted 23 June 2008 